\title{
Thermophysical properties of the lanthanide sesquisulfides. I. Schottky functions and magnetic and electronic properties of $\gamma-\mathrm{La}_{2} \mathrm{~S}_{3}, \gamma-\mathrm{Ce}_{2} \mathrm{~S}_{3}$, $\gamma-\mathrm{Nd}_{2} \mathrm{~S}_{3}$, and $\gamma-\mathrm{Gd}_{2} \mathrm{~S}_{3}$
}

\author{
Edgar F. Westrum, Jr. and Ramón Burriel a) \\ Department of Chemistry, University of Michigan, Ann Arbor, Michigan 48109 \\ John B. Gruber' \\ Department of Physics, San Jose State University, San Jose, California 95192 \\ P. E. Palmer and B. J. Beaudry \\ Ames Laboratory, Iowa State University, Ames, Iowa 50011
}

W. A. Plautz ${ }^{\text {) }}$

School of Medicine, Washington University, St. Louis, Missouri 63110

(Received 23 February 1989; accepted 14 July 1989)

\begin{abstract}
Heat capacity measurements of four lanthanide sesquisulfides $\mathrm{La}_{2} \mathrm{~S}_{3}, \mathrm{Ce}_{2} \mathrm{~S}_{3}, \mathrm{Nd}_{2} \mathrm{~S}_{3}$, and $\mathrm{Gd}_{2} \mathrm{~S}_{3}$, prepared in the $\gamma$ phase, have been obtained between 6 and $350 \mathrm{~K}$ by adiabatic calorimetry. The total heat capacity has been resolved into lattice, electronic, magnetic, and Schottky components. The Schottky contributions agree well with the calculated values based on the observed splitting of the ground-state manifold of the rare earth ions occupying sites of $\mathrm{S}_{4}$ symmetry in the $\mathrm{Th}_{3} \mathrm{P}_{4}$ structure. The observed splitting is obtained from an analysis of the hot bands in the absorption spectrum and from direct observation of the Stark levels in the far infrared. The Stark levels (all doublets) for $\mathrm{Ce}_{2} \mathrm{~S}_{3}\left({ }^{2} F_{5 / 2}\right)$ are 0,185 , and $358 \mathrm{~cm}^{-1}$; for $\mathrm{Nd}_{2} \mathrm{~S}_{3}\left({ }^{4} I_{9 / 2}\right)$, they are $0,76,150,180$, and $385 \mathrm{~cm}^{-1}$. For $\mathrm{La}_{2} \mathrm{~S}_{3}$, which has no Schottky or magnetic contributions to the heat capacity, the thermal data can be extrapolated to $0 \mathrm{~K}$. The entropy for $\mathrm{La}_{2} \mathrm{~S}_{3}$ at $298.15 \mathrm{~K}$ (as $S^{\circ} / R$ ) is 19.51 . Schottky and magnetic ordering at lower temperatures in $\mathrm{Ce}_{2} \mathrm{~S}_{3}, \mathrm{Nd}_{2} \mathrm{~S}_{3}$, and $\mathrm{Gd}_{2} \mathrm{~S}_{3}$ preclude such extrapolation techniques. Therefore the entropy at $298.15 \mathrm{~K}$ for these compounds $\left\{S^{0}-S^{0}(7 \mathrm{~K})\right\} / R$, is $21.34,22.38$, and 20.05, respectively.
\end{abstract}

\section{INTRODUCTION}

Lanthanide sesquisulfides $\left(\operatorname{Ln}_{2} S_{3}\right)$-as a consequence of their $4 f^{n}$ states-manifest unusual magnetic and electronic behavior. ${ }^{1-21}$ The $4 f$ electrons are localized in the inner shell of the lanthanide ions, but the polarization of the $5 d$ conduction electrons is known to give ferro- and antiferromagnetic interactions in lanthanide alloys due to indirect exchange of the RKKY type. Moreover, the $\mathrm{Th}_{3} \mathbf{P}_{4}$ structure, to which the $\gamma$ phase of the lanthanide sesquisulfides belongs, allows considerable variation of the composition. One-ninth of the positions of the $\mathrm{Ln}$ cation are randomly unoccupied in $\mathrm{Ln}_{2} \mathrm{~S}_{3}$. They can be filled with excess $\mathrm{Ln}$ up to the composition $\mathrm{Ln}_{3} \mathrm{~S}_{4}$ thereby providing additional free electrons that profoundly change the physical properties of the compound. This property makes them suitable materials in which the number of conduction electrons can be adjusted at will to the required physical properties. One finds a whole range of ferro- and antiferromagnetic ordering, insulators, semiconductors, and superconductors associated with these materials. They are found as components in solar energy absorbers, in efficient solar photovoltaic cells, or in infrared parametric oscillators. ${ }^{2,7,20}$

\footnotetext{
a) Present address: Instituto de Ciencia de los Materiales de Aragón, Departamento de Termología, Universidad de Zaragoza, 50009-Zaragoza, Spain.

b) Fellow, American Society for Engineering Education.

c) Work performed at the University of Michigan, Ann Arbor, MI.
}

The materials we study in this series of papers belong to the limiting composition $\operatorname{Ln}_{2} \mathrm{~S}_{3}$, in which, ideally, there are no nonlocalized electrons. They are insulators and we can extract the physical properties that depend on the lattice structure and single ion properties. The crystal field surrounding the lanthanide ions acts as a small perturbation on the localized electrons. The field splits the ground state into sublevels called Stark levels. Hence, thermophysical effects are expected when the higher-energy Stark levels of the lanthanide ions are depopulated on lowering the temperature. Specifically, the heat capacity shows Schottky contributions manifesting the magnitude of the splitting and degeneracy of the Stark levels of the ground state. These phenomena revealed by calorimetric, ${ }^{5-18}$ spectroscopic, ${ }^{1-4,19}$ and magnetic techniques ${ }^{13,15,20,21}$ provide elucidation and resolution of the energetic spectra. The infrared and Raman spectra, the magnetic susceptibility, and analysis of the crystal-field splitting of these compounds enable one to make an interpretation of the observed heat-capacity values and thermophysical functions, and resolved Schottky contributions. Moreover, subsequent comparison made with crystal-field lattice-sum calculations provides further evidence of the essential validity of this interpretation of the entire gamut of the thermophysical, crystalline electric field (CEF), and related properties as has already been done for the bixbyite sesquioxides. $^{22}$

Subambient heat-capacity determinations represent an important technique for study of these materials and their 
Schottky and magnetic contributions. Either of them may be resolved even at moderate temperatures by an essentially volumetric scheme for the estimation of lattice heat capacities. ${ }^{23,24}$

Heat-capacity data on lanthanide sesquisulfides of different preparations, compositions, and crystal structures obtained between 0.3 and $20 \mathrm{~K}$ are available in the literature. $^{7-13}$ Systematic studies also exist for the lanthanide monosulfides and for the $\mathrm{Ln}_{3} \mathrm{~S}_{4}$ compounds, but are of relevance here in only a few respects. ${ }^{6,15,25,26}$ Heat capacities of $\mathrm{Ln}_{3} \mathrm{Ch}_{4}(\mathrm{Ch}=\mathrm{Se}, \mathrm{Te}$, etc.) and sesquisulfide systems (e.g., mixed rare earth with $\mathrm{S}, \mathrm{Se}$, or $\mathrm{Te}$ ), and the binary systems $\mathrm{Ln}_{3} \mathrm{Ch}_{4}-\mathrm{Ln}_{2} \mathrm{Ch}_{3}$ have interesting thermophysical properties. For example, $T_{c}$ in $\mathrm{La}_{3} \mathrm{~S}_{4}(7 \mathrm{~K})$ drops essentially to $0 \mathrm{~K}$ in $\mathrm{La}_{2} \mathrm{~S}_{3}$. In fact, treatment of the lanthanide sulfides $\left(\mathrm{LnS}_{x}\right)$ as a homologous series provides useful correlation of thermophysical values for device applications. ${ }^{27}$

Many of the heat capacity values reported for $\operatorname{Ln}_{2} S_{3}$ do not extend to temperatures sufficiently high to enable one to determine the Schottky contribution due to the crystal-field splitting of the ground-state manifold of the tripositive rare earth ion. Furthermore, the samples investigated were not well characterized. Our purpose here is to detail stoichiometric sample preparation and crystal structure of the $\operatorname{Ln}_{2} \mathrm{~S}_{3}$ compounds we investigated. We report heat capacity measurements between 6 and $350 \mathrm{~K}$ which can be resolved into lattice, magnetic, and Schottky components. In this first paper, we compare the experimental Schottky contribution obtained for $\mathrm{Ce}_{2} \mathrm{~S}_{3}$ and $\mathrm{Nd}_{2} \mathrm{~S}_{3}$ with the calculated contribution based on the crystal-field splitting of the ground-state manifold as deduced from optical spectra. Overall agreement indicates that the volumetric method of analysis described ear$\operatorname{lier}^{23,24}$ is a useful approach to these systems.

\section{EXPERIMENTAL}

\section{A. Sample provenance and characterization for heat- capacity studies}

The four sulfide samples were prepared at the Ames Laboratory by direct combination of the pure elements in a manner similar to that described by Gschneidner, et al. ${ }^{11}$ The rare earth metals were also prepared in the Ames Laborator ${ }^{28}$ and had the chemical analyses typical of the highly purified metals reported earlier. ${ }^{28}$ Sublimed sulfur (99.999\%) was obtained from ASARCO. ${ }^{29}$ After synthesis of $\operatorname{Ln}_{2} S_{3}$ was achieved in sealed quartz ampoules, the samples were not melted as they had been in a previous study. ${ }^{14}$
Instead, the quartz ampoules were opened and the sulfide ground and sieved to 200 mesh powder. The powder was cold pressed into pellets $4 \mathrm{~mm}$ thick by $15 \mathrm{~mm}$ diameter. The pressed pellets of $\mathrm{La}_{2} \mathrm{~S}_{3}, \mathrm{Ce}_{2} \mathrm{~S}_{3}$, and $\mathrm{Nd}_{2} \mathrm{~S}_{3}$ were heated to $1500^{\circ} \mathrm{C}$ under a dynamic $\mathrm{H}_{2} \mathrm{~S}$ atmosphere for $48 \mathrm{~h}$. The $\mathrm{Gd}_{2} \mathrm{~S}_{3}$ was heated to $1450{ }^{\circ} \mathrm{C}$ for $24 \mathrm{~h}$ under dynamic $\mathrm{H}_{2} \mathrm{~S}$ treatment.

Debye Scherrer $x$-ray patterns contained only lines of the $\gamma$-phase bcc structure of $\mathrm{Th}_{3} \mathrm{P}_{4}$. Lattice parameters determined in this study are given in Table I along with the literature values. ${ }^{30}$ The final compositions were determined by G. V. Austin and R. Z. Bachman, Analytical Services Group, Ames Laboratory, Iowa State University, using the following procedures: the sesquisulfides were dissolved in 4.8 $\mathrm{M}$ hydrochloric acid and the evolved hydrogen sulfide gas was collected in a solution of sodium hydroxide and hydrogen peroxide. The sulfate which formed was determined gravimetrically as barium sulfate. The lanthanide cations in solution were quantitatively analyzed by titrating with EDTA. A sensitive test used to determine the presence of trace oxysulfide impurities was performed by dissolving the sulfide in $6 \mathrm{~N} \mathrm{HCl}$ and observing the clarity of the solution. All samples used in this study gave clear solutions when dissolved; this showed the absence of oxysulfides. ${ }^{30}$

\section{B. Automated adiabatic calorimetry}

The data were taken in the Mark X calorimetric cryostat, an improved (by addition of a guard shield surrounding the adiabatic shield $)^{31}$ version of the Mark II cryostat previously described together with the relevant operating techniques. The acquisition of heat-capacity data from about 6 to $350 \mathrm{~K}$ was computer assisted ${ }^{32}$ After being programmed for a series of determinations, the computer recorded: (i) drift readings of the calorimeter temperature (including the first and second derivatives) over a suitable length of time before and after each energy input to determine the equilibrium temperature and drift; and (ii) during each energy input, current and potential of the heater and the duration of the heating period. Information was recorded on the initial, final, and mean temperatures, on the energy input, and on the resistance of the heater, together with the apparent heat capacity of the system (including the calorimeter, heater, thermometer, and sample).

A gold-plated, oxygen-free, high-conductivity copper calorimeter (laboratory designation W-61) especially equipped with a pair of perforated copper spring-loaded sleeves soldered to the heater-thermometer well to hold the

TABLE I. Lattice parameters and analyzed compositions of the $\gamma$-lanthanide sulfides used in this study.

\begin{tabular}{|c|c|c|c|c|c|c|}
\hline \multirow[b]{2}{*}{ Sesquisulfide } & \multicolumn{2}{|c|}{ Lattice parameter $(\AA)$} & \multirow{2}{*}{$\begin{array}{l}\text { Analyzed mole ratio } \\
\text { S/Ln }\end{array}$} & \multirow[b]{2}{*}{$\mathrm{m} / \mathrm{g}$} & \multirow[b]{2}{*}{$\mathrm{M} / \mathrm{g} \mathrm{mol}^{-1}$} & \multirow[b]{2}{*}{ Color } \\
\hline & Present study & Reference 30 & & & & \\
\hline $\mathbf{L a}_{2} \mathbf{S}_{3}$ & $8.7220 \pm 0.0007$ & 8.731 & $1.497 \pm 0.005$ & 37.8289 & 374.003 & Yellow-green \\
\hline $\mathrm{Ce}_{2} \mathrm{~S}_{3}$ & $8.636 \pm 0.0002$ & 8.630 & $1.500 \pm 0.001$ & 38.1789 & 376.432 & Dark gray \\
\hline $\mathrm{Nd}_{2} \mathrm{~S}_{3}$ & $8.5248 \pm 0.0002$ & 8.527 & $1.500 \pm 0.006$ & 36.3252 & 384.66 & Yellow-blue \\
\hline $\mathrm{Gd}_{2} \mathrm{~S}_{3}$ & $8.3779 \pm 0.0003$ & 8.387 & $1.503 \pm 0.003$ & 36.1260 & 410.692 & Yellow \\
\hline
\end{tabular}


sulfide pellets was employed for all measurements. After loading, the calorimeter was evacuated and about $2.0 \mathrm{kPa}$ (at $300 \mathrm{~K}$ ) helium gas added to facilitate thermal equilibration. The calorimeter seal-off tip was closed with Cerroseal (50 mass \% tin and indium) solder. Relevant adjuvant quantities (sample mass, molar masses, lattice constants, etc.) used in computer reduction of the data are also given in Table I. The mole used throughout this paper is based on the formula written as $\mathrm{Ln}_{2} \mathrm{~S}_{3}$ and thus involves 2 mol of lanthanide metal. Temperatures were measured on an ASL-7 induction bridge with a Leeds and Northrup platinum-resistance thermometer sensor calibrated by the National Institute of Standards and Technology. All other accurate experimental quantities are referred to standards and calibrations provided by NIST.

\section{Optical spectroscopy: Samples and technique}

The spectra of $\gamma$-phase single crystal $\mathrm{Ce}_{2} \mathrm{~S}_{3}$ and $\mathrm{Nd}_{2} \mathrm{~S}_{3}$ were first observed by Henderson et al. ${ }^{1,2}$ They prepared the material by passing purified $\mathrm{H}_{2} \mathrm{~S}$ over finely divided oxide powder in graphite boats at temperatures between 850 and $1300^{\circ} \mathrm{C}$. The powders were used to grow single crystals by the Bridgman method. ' The $\mathrm{x}$-ray crystallographic patterns of pieces of the crystals indicated that $\mathrm{Ce}_{2} \mathrm{~S}_{3}$ and $\mathrm{Nd}_{2} \mathrm{~S}_{3}$ were grown in the $\gamma$ phase.
Chemical analysis of the crystals indicated the materials were stoichiometric $\left(\mathrm{LnS}_{1.500 \pm 0.002}\right) .^{1-4}$

Infrared spectra on powdered mull samples of $\mathrm{La}_{2} \mathrm{~S}_{3}$, $\mathrm{Ce}_{2} \mathrm{~S}_{3}$, and $\mathrm{Nd}_{2} \mathrm{~S}_{3}$ were recorded between 2.5 and $40 \mu$ using a Perkin-Elmer 301 spectrophotometer. The infrared spectra between 10 and $200 \mu$ of single crystals were recorded using a Beckman FS-720 Fourier interference spectrophotometer. Samples were cooled to 15 and $90 \mathrm{~K}$ using a conduction dewar filled sequentially with liquid helium and liquid nitrogen. The measurements were performed by Henderson, Muramoto, and Gruber. ${ }^{1-3}$

\section{RESULTS}

\section{A. Calorimetric determinations}

The values for the heat capacity of $\mathrm{La}_{2} \mathrm{~S}_{3}$ presented in Table II in energy-dimensionless units are in chronological sequence, so that the temperature increments can usually be deduced from the sequential temperatures. Corresponding values for the other three compounds are given in Table II. The data were smoothed at the lowest temperatures by use of a $C_{p} / R T$ vs $T^{2}$ plot. From such a plot, we obtained for $\mathrm{La}_{2} \mathrm{~S}_{3}$ a Debye temperature $\theta_{0}=284 \mathrm{~K}$, and an electronic coefficient $\gamma=0 \pm 0.0002 R K$. The temperature range of our measurements did not permit the estimation of a more accurate value for $\gamma$.

TABLE II. Molar heat capacities of lanthanide sesquisulfides $\left(R=8.3144 \mathrm{~J} \mathrm{~K}^{-1} \mathrm{~mol}^{-1}\right)$.

\begin{tabular}{|c|c|c|c|c|c|c|c|}
\hline$T(\mathbf{K})$ & $C_{p} / R$ & $T(\mathrm{~K})$ & $C_{p} / R$ & $T(\mathbf{K})$ & $C_{p} / R$ & $T(\mathrm{~K})$ & $C_{p} / R$ \\
\hline \multicolumn{8}{|c|}{$\begin{array}{l}\text { Lanthanum } \\
\text { sesquisulfide }\left(\mathrm{La}_{2} \mathrm{~S}_{3}\right)\end{array}$} \\
\hline & & Series II & & Series IV & & 19.72 & 0.493 \\
\hline Series I & $12521^{\mathrm{a}}$ & 71.98 & 6.406 & 40.93 & 2.759 & 21.43 & 0.630 \\
\hline 180.08 & $12.521^{\circ}$ & 74.77 & 6.710 & 42.57 & 2.926 & 23.33 & 0.780 \\
\hline 185.09 & 12.735 & 78.14 & 7.032 & 44.62 & 3.198 & 25.31 & 0.976 \\
\hline 190.23 & 12.847 & 81.66 & 7.425 & 46.75 & 3.456 & 27.14 & 1.171 \\
\hline 195.38 & 12.969 & 85.69 & 7.802 & 48.98 & 3.732 & 28.93 & 1.355 \\
\hline 200.54 & 13.091 & 89.94 & 8.207 & 51.32 & 4.016 & 31.00 & 1.579 \\
\hline 205.70 & 13.169 & 94.41 & 8.543 & 53.79 & 4.320 & 33.32 & 1.845 \\
\hline 210.86 & 13.252 & $\begin{array}{l}94.41 \\
99.12\end{array}$ & 8.907 & 56.38 & 4.626 & 35.90 & 2.149 \\
\hline 216.02 & 13.385 & 104.03 & 9.293 & 59.10 & 4.963 & 38.76 & 2.489 \\
\hline 223.76 & 13.493 & 122.14 & 10.439 & 61.96 & 5.302 & 41.94 & 2.862 \\
\hline 228.88 & 13.560 & 129.82 & 10.854 & 64.97 & 5.673 & & \\
\hline 234.03 & 13.638 & 134.93 & 11.081 & 68.13 & 5.999 & \multicolumn{2}{|c|}{ Cerium sesquisulfide $\left(\mathrm{Ce}_{2} \mathrm{~S}_{3}\right)$} \\
\hline 239.20 & 13.715 & 140.01 & 11.293 & 71.47 & 6.359 & & \\
\hline 244.38 & 13.770 & 145.11 & 11.510 & 74.96 & 6.732 & & \\
\hline 249.55 & 13.836 & 150.21 & 11.722 & 78.65 & 7.115 & Series I & \\
\hline 254.73 & 13.896 & 155.32 & 11.919 & 82.52 & 7.521 & 148.74 & 12.896 \\
\hline 259.91 & 13.915 & 160.26 & 12.083 & & & 153.68 & 13.088 \\
\hline 265.08 & 13.986 & 165.22 & 12.274 & Series V & & 158.81 & 13.251 \\
\hline 270.26 & 14.070 & 170.37 & 12.350 & 65.71 & 5.751 & 163.92 & 13.384 \\
\hline 275.44 & 14.091 & 175.51 & 12.528 & 69.02 & 6.094 & 169.03 & 13.514 \\
\hline 282.03 & 14.154 & 180.65 & 12.663 & 72.38 & 6.457 & 174.17 & 13.658 \\
\hline 287.20 & 14.202 & 185.81 & 12.750 & 75.94 & 6.759 & 179.31 & 13.786 \\
\hline 292.38 & 14.254 & 190.95 & 12.893 & 83.59 & 7.611 & 184.46 & 13.876 \\
\hline 297.57 & 14.279 & 196.11 & 12.991 & & & 189.61 & 13.990 \\
\hline 302.76 & 14.301 & & & Series VI & & 194.78 & 14.080 \\
\hline 307.94 & 14.361 & Series III & & 7.72 & 0.022 & 199.95 & 14.164 \\
\hline 313.12 & 14.415 & 105.22 & 9.357 & 8.30 & 0.029 & 205.11 & 14.232 \\
\hline 318.30 & 14.489 & 110.25 & 9.709 & 9.11 & 0.038 & 210.27 & 14.293 \\
\hline 323.49 & 14.533 & 121.12 & 10.378 & 10.35 & 0.057 & 215.42 & 14.370 \\
\hline 328.68 & 14.564 & 126.10 & 10.645 & 12.76 & 0.116 & 220.59 & 14.441 \\
\hline 333.87 & 14.598 & 131.18 & 10.894 & 13.71 & 0.150 & 225.76 & 14.517 \\
\hline 339.06 & 14.611 & 136.26 & 11.153 & 14.81 & 0.198 & 230.94 & 14.549 \\
\hline 344.25 & 14.660 & 141.35 & 11.362 & 18.19 & 0.386 & 236.12 & 14.625 \\
\hline
\end{tabular}


TABLE II (continued).

\begin{tabular}{|c|c|c|c|c|c|c|c|}
\hline$T(\mathbf{K})$ & $C_{p} / R$ & $T(\mathbf{K})$ & $C_{p} / R$ & $T(\mathbf{K})$ & $C_{p} / R$ & $T(\mathbf{K})$ & $C_{p} / R$ \\
\hline 241.19 & 14.640 & 36.84 & 2.423 & 10.28 & 0.144 & Series I & \\
\hline 246.37 & 14.732 & 38.89 & 2.687 & 10.77 & 0.149 & 56.64 & 4.666 \\
\hline 251.66 & 14.770 & 41.20 & 2.992 & 11.27 & 0.164 & 59.53 & 4.972 \\
\hline 256.84 & 14.785 & 43.78 & 3.334 & 11.80 & 0.176 & 62.42 & 5.285 \\
\hline 262.02 & 14.834 & 46.38 & 3.691 & 12.35 & 0.196 & 65.45 & 5.617 \\
\hline 267.19 & 14.864 & 51.37 & 4.364 & 12.92 & 0.218 & 68.64 & 5.928 \\
\hline 272.36 & 14.885 & 53.39 & 4.628 & 13.52 & 0.248 & 72.01 & 6.253 \\
\hline 277.52 & 14.933 & 56.28 & 5.025 & 14.09 & 0.278 & 75.54 & 6.598 \\
\hline 282.71 & 14.948 & 59.15 & 5.436 & 14.69 & 0.319 & 79.26 & 6.980 \\
\hline 287.89 & 14.983 & 62.05 & 5.834 & 15.36 & 0.367 & 83.16 & 7.346 \\
\hline 293.05 & 14.994 & 65.42 & 6.289 & 16.06 & 0.422 & 87.27 & 7.737 \\
\hline 298.16 & 14.989 & 69.28 & 6.773 & 16.79 & 0.486 & 91.59 & 8.099 \\
\hline 303.33 & 15.047 & 73.14 & 7.246 & 17.55 & 0.558 & 95.90 & 8.404 \\
\hline 308.50 & 15.045 & 77.04 & 7.709 & 18.35 & 0.641 & 100.49 & 8.741 \\
\hline 313.70 & 15.080 & \multirow{3}{*}{\multicolumn{2}{|c|}{$\begin{array}{l}\text { Neodymium sesquisulfide } \\
\left(\mathrm{Nd}_{2} \mathrm{~S}_{3}\right)\end{array}$}} & 19.18 & 0.733 & 105.50 & 9.099 \\
\hline 318.91 & 15.114 & & & 20.06 & 0.837 & 110.51 & 9.419 \\
\hline 324.10 & 15.138 & & & 20.97 & 0.955 & 115.54 & 9.765 \\
\hline 329.28 & 15.186 & Series I & & 21.93 & 1.084 & 120.59 & 10.032 \\
\hline 334.47 & 15.225 & 117.69 & 11.270 & 22.93 & 1.225 & 125.66 & 10.341 \\
\hline 339.65 & 15.251 & 121.32 & 11.449 & 23.99 & 1.380 & 130.72 & 10.566 \\
\hline 344.84 & 15.289 & 125.12 & 11.633 & 25.09 & 1.549 & 135.79 & 10.830 \\
\hline \multirow[t]{2}{*}{348.76} & 15.315 & 130.14 & 11.840 & 26.25 & 1.726 & 140.88 & 11.094 \\
\hline & & 135.23 & 12.069 & 27.47 & 1.925 & 145.98 & 11.316 \\
\hline Series II & & 140.33 & 12.255 & 28.67 & 2.119 & 151.09 & 11.530 \\
\hline 76.68 & 7.659 & 145.44 & 12.447 & 29.94 & 2.321 & 156.21 & 11.714 \\
\hline 80.05 & 8.075 & 150.56 & 12.622 & 31.34 & 2.555 & 161.34 & 11.866 \\
\hline 84.00 & 8.535 & 155.68 & 12.764 & 32.81 & 2.792 & 166.47 & 12.042 \\
\hline 88.15 & 8.992 & 160.81 & 12.929 & 34.36 & 3.053 & 171.60 & 12.210 \\
\hline 92.53 & 9.393 & 165.95 & 13.034 & 35.98 & 3.313 & 176.74 & 12.344 \\
\hline 97.15 & 9.777 & 171.08 & 13.160 & 37.68 & 3.585 & 181.88 & 12.483 \\
\hline 102.00 & 10.188 & 176.19 & 13.273 & 39.47 & 3.864 & 187.04 & 12.622 \\
\hline 106.99 & 10.575 & 181.31 & 13.366 & 41.35 & 4.146 & 192.19 & 12.732 \\
\hline 112.02 & 10.941 & 186.46 & 13.468 & 43.32 & 4.465 & 197.35 & 12.859 \\
\hline 117.06 & 11.304 & 191.62 & 13.556 & 45.40 & 4.752 & 202.51 & 12.943 \\
\hline 122.11 & 11.607 & 196.78 & 13.638 & 47.59 & 5.069 & 207.67 & 13.052 \\
\hline 127.18 & 11.912 & 201.94 & 13.706 & 49.88 & 5.373 & 212.83 & 13.169 \\
\hline 137.66 & 12.450 & 207.10 & 13.784 & 52.29 & 5.697 & 217.99 & 13.254 \\
\hline 142.71 & 12.678 & 212.27 & 13.838 & 54.83 & 6.015 & 223.16 & 13.358 \\
\hline 147.82 & 12.889 & 217.44 & 13.927 & 57.50 & 6.351 & 228.33 & 13.422 \\
\hline \multirow[t]{2}{*}{152.94} & 13.074 & 222.62 & 13.987 & 60.30 & 6.695 & 233.50 & 13.513 \\
\hline & & 227.80 & 14.046 & 63.25 & 7.044 & 238.68 & 13.588 \\
\hline Series III & & 232.96 & 14.119 & 66.35 & 7.383 & 243.85 & 13.679 \\
\hline 6.84 & 0.163 & 238.13 & 14.142 & & & 249.02 & 13.724 \\
\hline 7.55 & 0.133 & 243.31 & 14.236 & Series III & & 254.20 & 13.787 \\
\hline 8.41 & 0.116 & 248.48 & 14.270 & 24.00 & 1.375 & 259.38 & 13.843 \\
\hline 9.18 & 0.111 & 253.66 & 14.321 & 39.79 & 3.918 & 264.55 & 13.906 \\
\hline 9.92 & 0.110 & 258.85 & 14.355 & 71.97 & 7.938 & 269.73 & 13.972 \\
\hline 10.64 & 0.116 & 264.03 & 14.405 & 75.44 & 8.279 & 274.90 & 14.011 \\
\hline 11.45 & 0.127 & 269.21 & 14.437 & 83.15 & 9.022 & 280.07 & 14.076 \\
\hline 12.35 & 0.146 & 274.39 & 14.486 & 87.18 & 9.381 & 285.23 & 14.107 \\
\hline 13.23 & 0.170 & 279.58 & 14.513 & 91.64 & 9.768 & & \\
\hline 14.10 & 0.202 & 284.76 & 14.545 & 96.65 & 10.056 & Series II & \\
\hline 14.98 & 0.241 & 289.93 & 14.593 & 101.66 & 10.368 & 276.68 & 14.020 \\
\hline 15.82 & 0.282 & 295.11 & 14.602 & 106.69 & 10.646 & 281.79 & 14.053 \\
\hline 16.68 & 0.328 & 300.28 & 14.648 & 111.72 & 10.926 & 286.88 & 14.110 \\
\hline 17.57 & 0.384 & 305.45 & 14.685 & 116.77 & 11.234 & 291.95 & 14.156 \\
\hline 18.46 & 0.445 & 310.62 & 14.707 & 117.51 & 11.261 & 297.01 & 14.207 \\
\hline 19.45 & 0.517 & 315.79 & 14.781 & 121.60 & 11.451 & 302.05 & 14.249 \\
\hline 20.53 & 0.600 & 320.95 & 14.797 & 125.73 & 11.651 & 307.08 & 14.291 \\
\hline 21.60 & 0.693 & 326.12 & 14.841 & 130.82 & 11.900 & 312.10 & 14.326 \\
\hline 22.77 & 0.802 & 331.29 & 14.904 & 135.92 & 12.113 & 317.13 & 14.382 \\
\hline 24.03 & 0.924 & 336.48 & 14.931 & 141.02 & 12.305 & 322.13 & 14.437 \\
\hline 25.29 & 1.052 & 341.66 & 14.985 & 146.15 & 12.478 & 327.11 & 14.501 \\
\hline 26.66 & 1.197 & 346.85 & $\begin{array}{l}14.903 \\
15.022\end{array}$ & 151.27 & 12.630 & 332.08 & 14.510 \\
\hline 28.11 & 1.357 & & & 156.41 & 12.791 & 337.03 & 14.543 \\
\hline 29.57 & 1.524 & Series II & & 161.54 & 12.931 & 342.03 & 14.612 \\
\hline 31.13 & 1.708 & $\begin{array}{c}\text { Series } 11 \\
8.24\end{array}$ & 0.138 & 166.68 & 13.050 & 346.91 & 14.663 \\
\hline 32.78 & 1.907 & $\begin{array}{l}8.24 \\
8.76\end{array}$ & $\begin{array}{l}0.130 \\
0.133\end{array}$ & 171.82 & 13.171 & & \\
\hline 33.54 & 2.001 & 9.29 & 0.135 & Gadolinium & uisulfide & Series III & \\
\hline 35.03 & 2.192 & 9.79 & 0.138 & $\left(G_{2} S_{3}\right)$ & juisuniae & 290.01 & 14.128 \\
\hline
\end{tabular}


TABLE II (continued).

\begin{tabular}{|c|c|c|c|c|c|c|c|}
\hline$T(\mathbf{K})$ & $C_{p} / R$ & $T(\mathbf{K})$ & $C_{p} / R$ & $T(\mathrm{~K})$ & $C_{p} / R$ & $T(\mathbf{K})$ & $C_{p} / R$ \\
\hline 295.06 & 14.186 & 291.72 & 14.156 & Series VII & & 24.10 & 1.134 \\
\hline 300.09 & 14.250 & 296.70 & 14.188 & 6.05 & 1.957 & 25.23 & 1.245 \\
\hline 305.10 & 14.272 & 301.67 & 14.229 & 6.36 & 1.791 & 26.49 & 1.369 \\
\hline 310.09 & 14.328 & 306.61 & 14.288 & 6.73 & 1.655 & 27.72 & 1.495 \\
\hline 315.08 & 14.382 & 314.00 & 14.358 & 7.14 & 1.347 & 29.02 & 1.621 \\
\hline 320.05 & 14.437 & 321.37 & 14.429 & 7.62 & 1.200 & 30.38 & 1.761 \\
\hline 325.00 & 14.461 & 326.26 & 14.468 & 8.16 & 1.063 & 31.80 & 1.928 \\
\hline 329.94 & 14.504 & 331.15 & 14.517 & 8.78 & 0.928 & 33.29 & 2.083 \\
\hline 334.88 & 14.543 & 336.03 & 14.554 & 9.45 & 0.824 & 34.86 & 2.269 \\
\hline 339.80 & 14.594 & 340.90 & 14.575 & 10.25 & 0.708 & 36.51 & 2.444 \\
\hline \multirow[t]{2}{*}{344.72} & 14.627 & 345.77 & 14.507 & 11.08 & 0.626 & 38.23 & 2.630 \\
\hline & & & & 11.97 & 0.579 & 40.05 & 2.833 \\
\hline Series IV & & Series VI & & 12.89 & 0.552 & 41.95 & 3.045 \\
\hline 323.05 & 14.424 & 6.02 & 2.037 & 13.80 & 0.540 & 43.95 & 3.260 \\
\hline 330.25 & 14.489 & 6.98 & 1.455 & 14.68 & 0.548 & 46.04 & 3.499 \\
\hline 337.44 & 14.556 & 8.45 & 0.976 & 15.59 & 0.570 & 48.25 & 3.740 \\
\hline 342.21 & 14.592 & 9.87 & 0.742 & 16.55 & 0.600 & 50.56 & 3.994 \\
\hline \multirow[t]{2}{*}{346.98} & 14.639 & 11.12 & 0.625 & 17.50 & 0.643 & 52.99 & 4.263 \\
\hline & & 12.26 & 0.568 & 18.44 & 0.695 & 55.54 & 4.533 \\
\hline Series V & & 13.32 & 0.543 & 19.39 & 0.753 & 58.23 & 4.813 \\
\hline 271.64 & 13.930 & 14.34 & 0.545 & 20.33 & 0.815 & 61.04 & 5.141 \\
\hline 276.69 & 13.991 & 15.32 & 0.564 & 21.27 & 0.891 & 64.00 & 5.476 \\
\hline 281.72 & 14.043 & 16.29 & 0.590 & 22.21 & 0.964 & 67.12 & 5.775 \\
\hline 286.73 & 14.078 & 17.24 & 0.629 & 23.15 & 1.045 & & \\
\hline
\end{tabular}

${ }^{a}$ This point not used in fitting curve.

The thermodynamic functions for $\mathrm{La}_{2} \mathrm{~S}_{3}$ are summarized in Table III with an occasional extra digit beyond those absolutely significant to facilitate interpolation. Corresponding values for the other three compounds are given in Table IV. It should be noted here that the data for the en- tropy, enthalpy, and Gibbs energy increments refer to $7 \mathrm{~K}$ rather than $0 \mathrm{~K}$. The experimental curves for the four compounds are depicted in Fig. 1. The presence of Schottky contributions to the heat capacities of $\mathrm{Nd}_{2} \mathrm{~S}_{3}$ and $\mathrm{Ce}_{2} \mathrm{~S}_{3}$ are quite evident upon comparison with $\mathrm{La}_{2} \mathrm{~S}_{3}$ and $\mathrm{Gd}_{2} \mathrm{~S}_{3}$ curves

TABLE III. Molar thermodynamic functions of lanthanum sesquisulfide $\left(R=8.3144 \mathrm{~J} \mathrm{~K}^{-1} \mathrm{~mol}^{-1}\right)$.

\begin{tabular}{|c|c|c|c|c|}
\hline$T(\mathrm{~K})$ & $C_{p} / R$ & $S^{0} / R$ & $\left\{H^{0}-H^{0}(0)\right\} / R K$ & $-\left\{G^{0}-H^{0}(0)\right\} / R T$ \\
\hline 0 & 0.0 & 0.0 & 0.0 & 0.0 \\
\hline 10 & 0.050 & 0.017 & 0.122 & 0.004 \\
\hline 15 & 0.206 & 0.061 & 0.698 & 0.015 \\
\hline 20 & 0.515 & 0.159 & 2.440 & 0.037 \\
\hline 25 & 0.948 & 0.319 & 6.054 & 0.077 \\
\hline 30 & 1.470 & 0.537 & 12.071 & 0.134 \\
\hline 40 & 2.638 & 1.117 & 32.531 & 0.304 \\
\hline 50 & 3.862 & 1.837 & 65.00 & 0.537 \\
\hline 60 & 5.065 & 2.648 & 109.68 & 0.820 \\
\hline 70 & 6.199 & 3.515 & 166.07 & 1.143 \\
\hline 80 & 7.236 & 4.412 & 233.33 & 1.495 \\
\hline 90 & 8.165 & 5.319 & 310.42 & 1.869 \\
\hline 100 & 8.983 & 6.222 & 396.25 & 2.260 \\
\hline 120 & 10.314 & 7.983 & 589.9 & 3.068 \\
\hline 140 & 11.309 & 9.652 & 806.6 & 3.891 \\
\hline 160 & 12.056 & 11.213 & 1040.6 & 4.709 \\
\hline 180 & 12.629 & 12.668 & 1287.7 & 5.514 \\
\hline 200 & 13.076 & 14.022 & 1544.9 & 6.298 \\
\hline 220 & 13.430 & 15.29 & 1810.1 & 7.058 \\
\hline 240 & 13.713 & 16.47 & 2081.6 & 7.793 \\
\hline 260 & 13.943 & 17.57 & 2358.3 & 8.504 \\
\hline 280 & 14.139 & 18.61 & 2639.1 & 9.189 \\
\hline 298.15 & 14.301 & 19.51 & 2897.2 & 9.790 \\
\hline 300 & 14.317 & 19.60 & 2923.7 & 9.850 \\
\hline 320 & 14.484 & 20.53 & 3211.7 & 10.489 \\
\hline 350 & 14.697 & 21.83 & 3649.6 & 11.406 \\
\hline
\end{tabular}


TABLE IV. Molar thermodynamic functions of lanthanide sesquisulfides $\left(R=8.3144 \mathrm{~J} \mathrm{~K}^{-1} \mathrm{~mol}^{-1}\right)$.

\begin{tabular}{|c|c|c|c|c|}
\hline$T(\mathbf{K})$ & $C_{p} / R$ & $\left\{S^{0}-S^{0}(7)\right\} / R$ & $\left\{H^{0}-H^{0}(7)\right\} / R K$ & $-\left\{G^{0}-H^{0}(7)\right\} / R T$ \\
\hline \multicolumn{5}{|c|}{ Cerium sesquisulfide $\left(\mathrm{Ce}_{2} \mathrm{~S}_{3}\right)$} \\
\hline 7 & 0.155 & 0.0 & 0.000 & 0.0 \\
\hline 10 & 0.110 & 0.044 & 0.363 & 0.007 \\
\hline 15 & 0.242 & 0.106 & 1.156 & 0.029 \\
\hline 20 & 0.559 & 0.215 & 3.088 & 0.061 \\
\hline 25 & 1.021 & 0.387 & 6.990 & 0.108 \\
\hline 30 & 1.575 & 0.621 & 13.451 & 0.173 \\
\hline 40 & 2.833 & 1.243 & 35.369 & 0.359 \\
\hline 50 & 4.174 & 2.019 & 70.40 & 0.611 \\
\hline 60 & 5.549 & 2.902 & 119.01 & 0.918 \\
\hline 70 & 6.867 & 3.858 & 181.17 & 1.269 \\
\hline 80 & 8.063 & 4.854 & 255.94 & 1.655 \\
\hline 90 & 9.116 & 5.866 & 341.95 & 2.066 \\
\hline 100 & 10.030 & 6.875 & 437.79 & 2.497 \\
\hline 120 & 11.488 & 8.839 & 653.7 & 3.392 \\
\hline 140 & 12.546 & 10.694 & 894.7 & 4.304 \\
\hline 160 & 13.288 & 12.421 & 1153.5 & 5.212 \\
\hline 180 & 13.795 & 14.017 & 1424.6 & 6.103 \\
\hline 200 & 14.153 & 15.49 & 1704.3 & 6.969 \\
\hline 220 & 14.431 & 16.85 & 1990.2 & 7.806 \\
\hline 240 & 14.656 & 18.12 & 2281.2 & 8.613 \\
\hline 260 & 14.822 & 19.30 & 2576.0 & 9.391 \\
\hline 280 & 14.932 & 20.40 & 2873.7 & 10.138 \\
\hline 298.15 & 15.01 & 21.34 & 3145.4 & 10.792 \\
\hline 300 & 15.02 & 21.43 & 3173.2 & 10.857 \\
\hline 320 & 15.12 & 22.41 & 3474.5 & 11.549 \\
\hline 350 & 15.33 & 23.77 & 3931.1 & 12.539 \\
\hline \multicolumn{5}{|c|}{ Neodymium sesquisulfide $\left(\mathrm{Nd}_{2} \mathrm{~S}_{3}\right)$} \\
\hline 7 & 0.134 & 0.0 & 0.000 & 0.0 \\
\hline 10 & 0.138 & 0.050 & 0.406 & 0.010 \\
\hline 15 & 0.340 & 0.134 & 1.472 & 0.036 \\
\hline 20 & 0.833 & 0.293 & 4.298 & 0.078 \\
\hline 25 & 1.532 & 0.551 & 10.143 & 0.145 \\
\hline 30 & 2.334 & 0.900 & 19.784 & 0.241 \\
\hline 40 & 3.946 & 1.796 & 51.28 & 0.513 \\
\hline 50 & 5.392 & 2.835 & 98.12 & 0.872 \\
\hline 60 & 6.660 & 3.933 & 158.53 & 1.290 \\
\hline 70 & 7.760 & 5.044 & 230.76 & 1.747 \\
\hline 80 & 8.709 & 6.144 & 313.23 & 2.228 \\
\hline 90 & 9.530 & 7.218 & 404.52 & 2.723 \\
\hline 100 & 10.240 & 8.260 & 503.5 & 3.225 \\
\hline 120 & 11.391 & 10.233 & 720.3 & 4.231 \\
\hline 140 & 12.253 & 12.057 & 957.2 & 5.220 \\
\hline 160 & 12.887 & 13.737 & 1208.9 & 6.181 \\
\hline 180 & 13.346 & 15.28 & 1471.5 & 7.108 \\
\hline 200 & 13.686 & 16.71 & 1741.9 & 7.998 \\
\hline 220 & 13.954 & 18.02 & 2018.4 & 8.850 \\
\hline 240 & 14.181 & 19.25 & 2299.8 & 9.666 \\
\hline 260 & 14.370 & 20.39 & 2585.4 & 10.448 \\
\hline 280 & 14.521 & 21.46 & 2874.4 & 11.197 \\
\hline 298.15 & 14.634 & 22.38 & 3139.0 & 11.850 \\
\hline 300 & 14.646 & 22.47 & 3166.0 & 11.915 \\
\hline 320 & 14.791 & 23.42 & 3460.3 & 12.604 \\
\hline 350 & 15.03 & 24.76 & 3908.1 & 13.589 \\
\hline \multicolumn{5}{|c|}{ Gadolinium sesquisulfide $\left(\mathrm{Gd}_{2} \mathrm{~S}_{3}\right)$} \\
\hline 7 & 1.445 & 0.0 & 0.000 & 0.0 \\
\hline 10 & 0.732 & 0.368 & 3.034 & 0.065 \\
\hline 15 & 0.555 & 0.608 & 5.969 & 0.211 \\
\hline 20 & 0.794 & 0.795 & 9.235 & 0.333 \\
\hline 25 & 1.219 & 1.015 & 14.215 & 0.447 \\
\hline 30 & 1.730 & 1.282 & 21.564 & 0.563 \\
\hline 40 & 2.829 & 1.929 & 44.333 & 0.821 \\
\hline 50 & 3.933 & 2.679 & 78.14 & 1.116 \\
\hline 60 & 5.025 & 3.493 & 122.96 & 1.444 \\
\hline 70 & 6.068 & 4.347 & 178.47 & 1.797 \\
\hline 80 & 7.038 & 5.221 & 244.08 & 2.170 \\
\hline 90 & 7.920 & 6.102 & 318.94 & 2.558 \\
\hline 100 & 8.712 & 6.978 & 402.18 & 2.957 \\
\hline
\end{tabular}


TABLE IV. (continued).

\begin{tabular}{lcccc}
\hline$T(\mathrm{~K})$ & $C_{p} / R$ & $\left\{S^{0}-S^{0}(7)\right\} / R$ & $\left\{H^{0}-H^{0}(7)\right\} / R K$ & $-\left\{G^{0}-H^{0}(7)\right\} / R T$ \\
\hline & & & & 3.770 \\
120 & 10.031 & 8.688 & 590.2 & 4.590 \\
140 & 11.048 & 10.315 & 801.4 & 5.402 \\
160 & 11.830 & 11.843 & 1030.5 & 6.198 \\
180 & 12.437 & 13.273 & 1273.4 & 6.973 \\
200 & 12.916 & 14.609 & 1527.2 & 7.725 \\
220 & 13.295 & 15.86 & 1789.4 & 8.452 \\
240 & 13.597 & 17.03 & 2058.4 & 9.154 \\
260 & 13.841 & 18.13 & 2332.9 & 9.833 \\
280 & 14.045 & 19.16 & 2611.8 & 10.428 \\
298.15 & 14.214 & 20.05 & 2868.3 & 10.487 \\
300 & 14.230 & 20.14 & 2894.6 & 11.120 \\
320 & 14.410 & 21.06 & 3181.0 & 12.028 \\
350 & 14.672 & 22.36 & 3617.3 & \\
\hline \hline
\end{tabular}

which are essentially pure lattice heat capacity over this range.

The accuracy of the data is characterized by $<0.06 \%$ standard deviation above $15 \mathrm{~K}$. Below this temperature, standard deviations of the heat capacity gradually increase until they attain an uncertainty of about $1 \%$ at the lowest temperatures.

\section{B. Schottky heat-capacity contributions}

Resolution of Schottky contributions from heat-capacity data on lanthanide compounds requires an accurate determination of the much larger "lattice" contribution. The volume-weighted lattice heat-capacity approximation technique has been used successfully in several other groups of lanthanide compounds (sesquioxides, halides, trihydroxides, etc. $)^{33-38}$ of the lanthanide sesquisulfides reported here, only $\mathrm{La}_{2} \mathrm{~S}_{3}$ has no excess contribution in the heat capacity. After subtraction of the low-temperature magnetic contribution in $\mathrm{Gd}_{2} \mathrm{~S}_{3}$, its lattice heat capacities can be used to interpolate the values for the other isostructural lanthanide sesquisulfides with the volumetric technique.

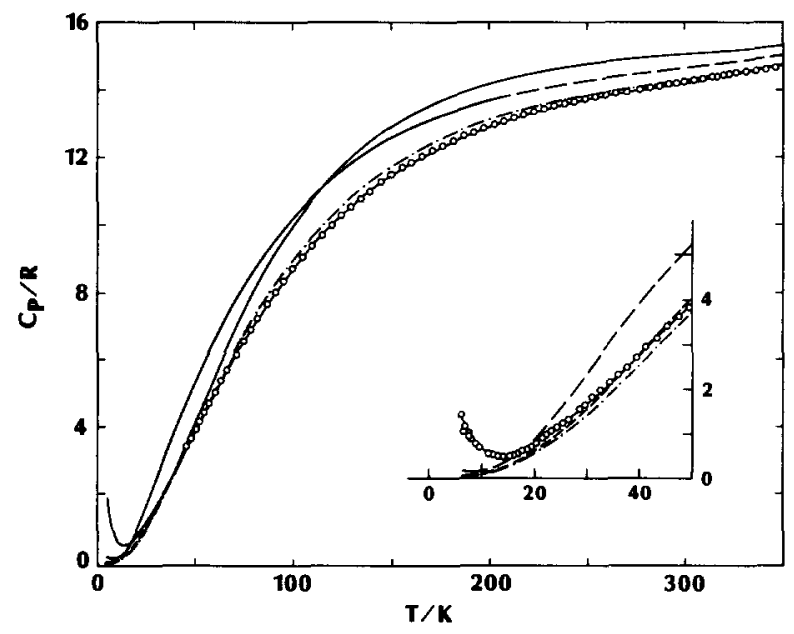

FIG. 1. Molar heat capacities of $\mathrm{La}_{2} \mathrm{~S}_{3}(-\cdot-\cdot) ; \mathrm{Ce}_{2} \mathrm{~S}_{3}(-)$, and (--); $\mathrm{Nd}_{2} \mathbf{S}_{3}(-,-,-)$; and $\mathrm{Gd}_{2} \mathbf{S}_{3}$ (-O-); experimental points shown for $\mathrm{Gd}_{2} \mathbf{S}_{3}$ to indicate typical density of data points obtained between 6 and $350 \mathrm{~K}$.
The calculation of $C_{v}^{L}(T)$ for $\mathrm{Gd}_{2} \mathrm{~S}_{3}$ below $50 \mathrm{~K}$ has been done by applying a corresponding-states principle to the heat capacity of $\mathrm{La}_{2} \mathrm{~S}_{3}$. This principle is followed quite accurately by isostructural compounds in which only one atom has been changed. Hence, their lattice heat capacities can be related by $C_{v}\left(\mathrm{Gd}_{2} \mathrm{~S}_{3}, T\right)=C_{v}\left(\mathrm{La}_{2} \mathrm{~S}_{3}, T / f\right)$.

The scaling factor $f=0.96$ has been determined in the temperature range where there are no anomalous contributions.

The other two sesquisulfides have broad Schottky anomalies in the whole temperature range, so that the scaling factor cannot be calculated in any temperature range.

Alternatively, the magnetic excess contribution is obtained by use of the single-parameter phonon-distribution approach of Komada and Westrum. ${ }^{39}$

The lattice heat capacities of $\gamma$-type lanthanide sesquisulfides may be expressed as follows:

$$
\begin{aligned}
C_{p}\left(\mathrm{Ce}_{2} \mathrm{~S}_{3}, \text { lattice }\right)= & \mathbf{x} C_{p}\left(\mathrm{Gd}_{2} \mathrm{~S}_{3}, \text { lattice }\right) \\
& +(1-\mathrm{x}) C_{p}\left(\mathrm{La}_{2} \mathrm{~S}_{3}\right),
\end{aligned}
$$

in which

$$
\mathbf{x}=\left\{\mathbf{V}\left(\mathrm{Ce}_{2} \mathrm{~S}_{3}\right)-\mathbf{V}\left(\mathrm{La}_{2} \mathrm{~S}_{3}\right)\right\} /\left\{\mathbf{V}\left(\mathrm{Gd}_{2} \mathrm{~S}_{3}\right)-\mathbf{V}\left(\mathrm{La}_{2} \mathrm{~S}_{3}\right)\right\}
$$

where $\mathbf{x}$ is the fractional increment in the molar volume $\mathbf{V}$. The qualification "lattice" attached to $\mathrm{Gd}_{2} \mathrm{~S}_{3}$ is a reminder that the cooperative magnetic contribution, insignificant above $50 \mathrm{~K}$, has been subtracted. The calorimetrically derived Schottky contributions to the heat capacities of $\mathrm{Ce}_{2} \mathrm{~S}_{3}$ and $\mathrm{Nd}_{2} \mathrm{~S}_{3}$ are displayed in the form of uninterrupted curves in Figs. 2 and 3.

\section{Optical spectroscopic determinations}

The infrared spectra between 2.5 and $40 \mu$ contain a number of strong bands which even at $15 \mathrm{~K}$ are considered to be broad $\left(20-25 \mathrm{~cm}^{-1}\right)$. There is a band with structure near $270 \mathrm{~cm}^{-1}$ in $\mathrm{La}_{2} \mathrm{~S}_{3}$ and $\mathrm{Ce}_{2} \mathrm{~S}_{3}$. A sharp, narrow band (halfmaximum of $8 \mathrm{~cm}^{-1}$ ) of moderate strength is found at approximately $358 \mathrm{~cm}^{-1}$ in $\mathrm{Ce}_{2} \mathrm{~S}_{3}$. This peak, which is not found in the $\mathrm{La}_{2} \mathrm{~S}_{3}$ spectrum, could be an electronic transition within the ground-state manifold ${ }^{2} F_{5 / 2}$ of the $\mathrm{Ce}^{3+}\left(4 f^{1}\right)$ ion. Since the point-group symmetry of $\mathrm{Ce}^{3+}$ in the $\mathrm{Ln}_{2} \mathrm{~S}_{3}$ 


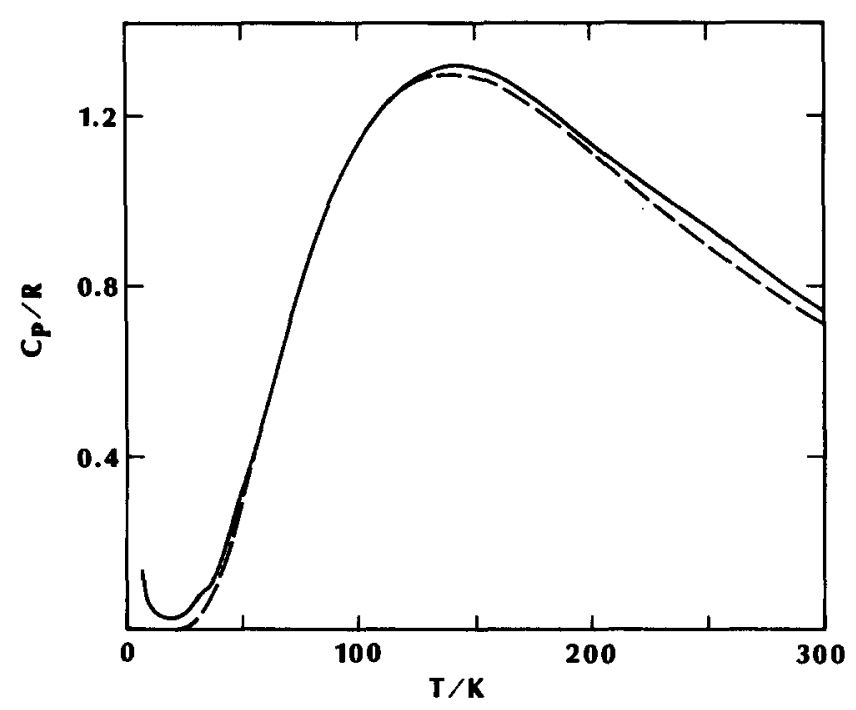

FIG. 2. Schottky contribution to the molar heat capacity of $\mathrm{Ce}_{2} \mathrm{~S}_{3}$; solid line represents calorimetric contribution $C_{p}\left(\mathrm{Ce}_{2} \mathrm{~S}_{3}\right)-\left[0.29 C_{p}\left(\mathrm{Gd}_{2} \mathrm{~S}_{3}\right)\right.$ lattice $+0.61 C_{\rho}\left(\mathrm{La}_{2} \mathrm{~S}_{3}\right)$ lattice $]$; dashed line represents contribution calculated from $\mathrm{Ce}^{3+}\left(4 f^{1}\right)^{2} F_{5 / 2}, 0(2), 185(2)$, and $358(2)$ in $\mathrm{cm}^{-1}$.

lattice is $S_{4}$, the ground-state manifold $J=5 / 2$ is split into three Kramers doublets. Earlier measurements and interpretation of magnetic susceptibility confirm that the ground-state Stark level is a doublet in $\mathrm{Ce}_{2} \mathrm{~S}_{3} \cdot{ }^{13}$ Absorption above $380 \mathrm{~cm}^{-1}$ is similar for $\mathrm{La}_{2} \mathrm{~S}_{3}$ and $\mathrm{Ce}_{2} \mathrm{~S}_{3}$. No analysis was made in the region around $2300 \mathrm{~cm}^{-1}$, where transitions to Stark levels of ${ }^{2} F_{7 / 2}$ are expected.

The infrared spectra of $\mathrm{La}_{2} \mathrm{~S}_{3}$ and $\mathrm{Ce}_{2} \mathrm{~S}_{3}$ (Fig. 4) were also investigated between 10 and $200 \mu$ with a Beckman FS-720 Fourier interference spectrophotometer using a conduction dewar filled with liquid helium. Bands approximately $20 \mathrm{~cm}^{-1}$ wide at half-maximum absorption were obtained at $60,120,170,230$, and $270 \mathrm{~cm}^{-1}$ for $\mathrm{La}_{2} \mathrm{~S}_{3}$ and 63,123 , 175,235 , and $275 \mathrm{~cm}^{-1}$ for $\mathrm{Ce}_{2} \mathrm{~S}_{3}$. The spectrum of $\mathrm{Ce}_{2} \mathrm{~S}_{3}$ at

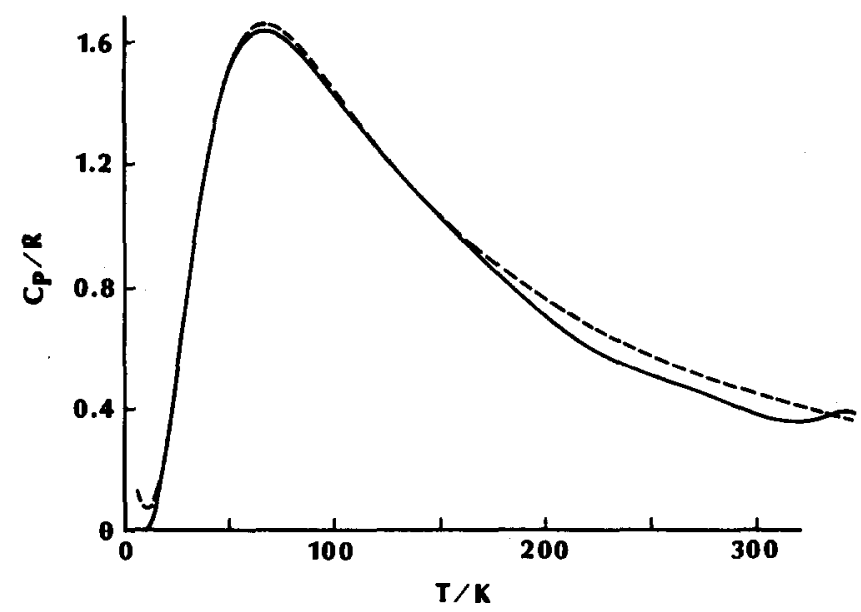

FIG. 3. Schottky contribution to the molar heat capacity of $\mathrm{Nd}_{2} \mathrm{~S}_{3}$; solid line represents calorimetric contribution $C_{\rho}\left(\mathrm{Nd}_{2} \mathrm{~S}_{3}\right)-\left[0.62 C_{p}\left(\mathrm{Gd}_{2} \mathrm{~S}_{3}\right)\right.$ lattice $+0.38 C_{\rho}\left(\mathrm{La}_{2} \mathrm{~S}_{3}\right)$ lattice]; dashed line represents contribution calculated from $\mathrm{Nd}^{3+}\left(4 f^{3}\right)^{4} I_{9 / 2}, \mathrm{O}(2), 76(2), 150(2), 180(2)$, and $385(2)$, in $\mathrm{cm}^{-1}$.

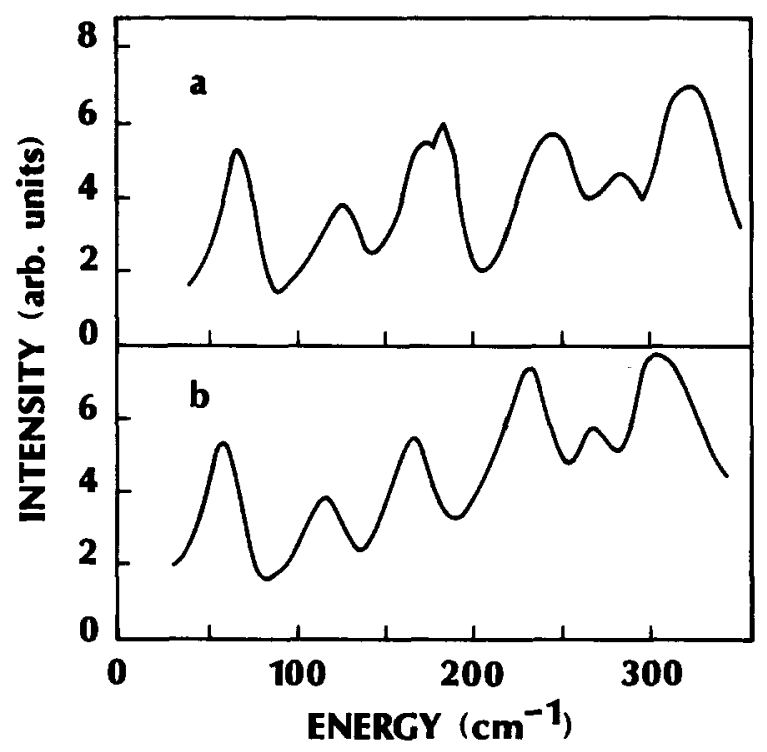

FIG. 4. Observed infrared absorption spectra of (a) $\mathrm{Ce}_{2} \mathrm{~S}_{3}$, and (b) $\mathrm{La}_{2} \mathrm{~S}_{3}$ obtained between 40 and $350 \mathrm{~cm}^{-1}$ at $15 \mathrm{~K}$; intensity is in arbitrary units.

$185 \mathrm{~cm}^{-1}$ shows a distinct shoulder not observed in the $\mathrm{La}_{2} \mathrm{~S}_{3}$ spectra which may be due to a second electronic transition within the ${ }^{2} F_{5 / 2}$ manifold. Otherwise, both spectra appear similar-except that the $\mathrm{Ce}_{2} \mathrm{~S}_{3}$ spectrum is shifted slightly to the high-energy side of the La spectrum.

Recent reports by various Russian groups, ${ }^{40,41}$ including the results of $300 \mathrm{~K}$ Raman scattering spectra of $\mathrm{La}_{2} \mathrm{~S}_{3}$, appear to be in agreement with an analysis of the vibrational spectra observed by Henderson, Muramoto, and Gruber. ${ }^{1,42}$

The absorption spectra of clear yellow-blue insulating crystals of $\mathrm{Nd}_{2} \mathrm{~S}_{3}$ recorded between 0.3 and $1.0 \mu$ at 300,80 , and $10 \mathrm{~K}$ were reported earlier by Henderson et al. ${ }^{4}$ Recently, the crystal-field splitting of the electronic $[S L] J$ energy levels of $\mathrm{Nd}^{3+}$ in $\mathrm{Nd}_{2} \mathrm{~S}_{3}$ was published along with a latticesum calculation for the crystal-field parameters. ${ }^{19}$ This paper makes use of the reported experimental Stark levels for the ground-state manifold $J=9 / 2\left({ }^{4} I_{9 / 2}\right)$ as well as the predicted splitting based on an effective point-charge model for the $\gamma-\mathrm{Nd}_{2} \mathrm{~S}_{3}$ lattice. In summary, Table $\mathrm{V}$ includes crystal-

TABLE V. Infrared and Raman spectra of $\operatorname{Ln}_{2} S_{3}$.

\begin{tabular}{|c|c|c|c|c|}
\hline \multicolumn{2}{|c|}{$\mathrm{La}_{2} \mathrm{~S}_{3}$} & \multirow{2}{*}{$\begin{array}{c}\mathrm{Ce}_{2} \mathrm{~S}_{3} \\
\mathrm{IR} \\
\left(\mathrm{cm}^{-1}\right)^{\mathrm{a}}\end{array}$} & \multicolumn{2}{|c|}{$\mathrm{Nd}_{2} \mathrm{~S}_{3}$} \\
\hline $\begin{array}{c}\text { IR } \\
\left(\mathrm{cm}^{-1}\right)^{\mathrm{a}}\end{array}$ & $\underset{\left(\mathrm{cm}^{-1}\right)^{b}}{\text { Raman }}$ & & $\frac{\text { IR }}{\left(\mathrm{cm}^{-1}\right)^{c}}$ & $\begin{array}{c}\text { Raman } \\
\left(\mathrm{cm}^{-1}\right)^{b}\end{array}$ \\
\hline 60 & $\cdots$ & 63 & 64 & $\cdots$ \\
\hline$\ldots$ & 85 & $\ldots$ & 76 & 80 \\
\hline $120,127^{b}$ & 122 & 123 & 124 & $\cdots$ \\
\hline$\ldots$ & $\cdots$ & $\cdots$ & 150 & $\cdots$ \\
\hline $170,180^{\mathrm{b}}$ & 185 & $175(185)$ & $180,200^{\mathrm{b}}$ & 192 \\
\hline $230,230^{\mathrm{b}}$ & 232 & 235 & $235,240^{\mathrm{b}}$ & 237 \\
\hline $270,270^{b}$ & 273 & 275 & $278^{b}$ & 287 \\
\hline $300,300^{b}$ & 300 & 310 & $\cdots$ & $\ldots$ \\
\hline . & $\cdots$ & 358 & 385 & $\cdots$ \\
\hline
\end{tabular}

Reference 42.

${ }^{6}$ References 41 and 40.

${ }^{\mathrm{C}}$ References 4 and 19. 
field doublet levels for $\mathrm{Nd}^{3+}$ at $0,76,150,180$, and $385 \mathrm{~cm}^{-1}$ and for $\mathrm{Ce}^{3+}$ at 0,185 , and $358 \mathrm{~cm}^{-1}$, along with the infrared and Raman spectra reported by Zhuze and his coworkers. ${ }^{40,41}$ Analysis of the vibronic spectra observed in the visible region agrees with the reported infrared and Raman spectra.

\section{DISCUSSION AND CONCLUSIONS}

Figure 5 compares the heat capacity data $\left(C_{p} / R\right)$ up to $20 \mathrm{~K}$ obtained in the present study with values reported by Ho et al. ${ }^{10}$ for $\gamma$ phase $\mathrm{La}_{2} \mathrm{~S}_{3}, \mathrm{Ce}_{2} \mathrm{~S}_{3}, \mathrm{Nd}_{2} \mathrm{~S}_{3}$, and $\mathrm{Gd}_{2} \mathrm{~S}_{3}$. Only in the instance of $\mathrm{La}_{2} \mathrm{~S}_{3}$ do our results tally closely. The range below $10 \mathrm{~K}$ is that over which we have the least accuracy; yet the consistency and smoothness of our values attest to the reproducibility of these values. Our agreement with Ho et al. ${ }^{10}$ for $\mathrm{La}_{2} \mathrm{~S}_{3}$ suggests concordance in calibration, etc. The $\mathrm{Ce}_{2} \mathrm{~S}_{3}$ trends are reasonably close, but in $\mathrm{Nd}_{2} \mathrm{~S}_{3}$ considerable divergence exists, and for $\mathrm{Gd}_{2} \mathrm{~S}_{3}$ our data suggest the presence of a transition with a peak below $7 \mathrm{~K}$. The differences in all instances are probably attributable to the high quality - with respect to both stoichiometry (cf. Table I, at worst $1.500 \pm 0.006$ ) and homogeneity- of the samples prepared for the present study in contrast with the dubiety of the earlier preparations. ${ }^{10}$

Another contrast concerns the data at higher temperatures in the subambient region as depicted on a deviation plot (Fig. 6). Deviation curves for literature values of $\mathrm{Nd}_{2} \mathrm{~S}_{3}$

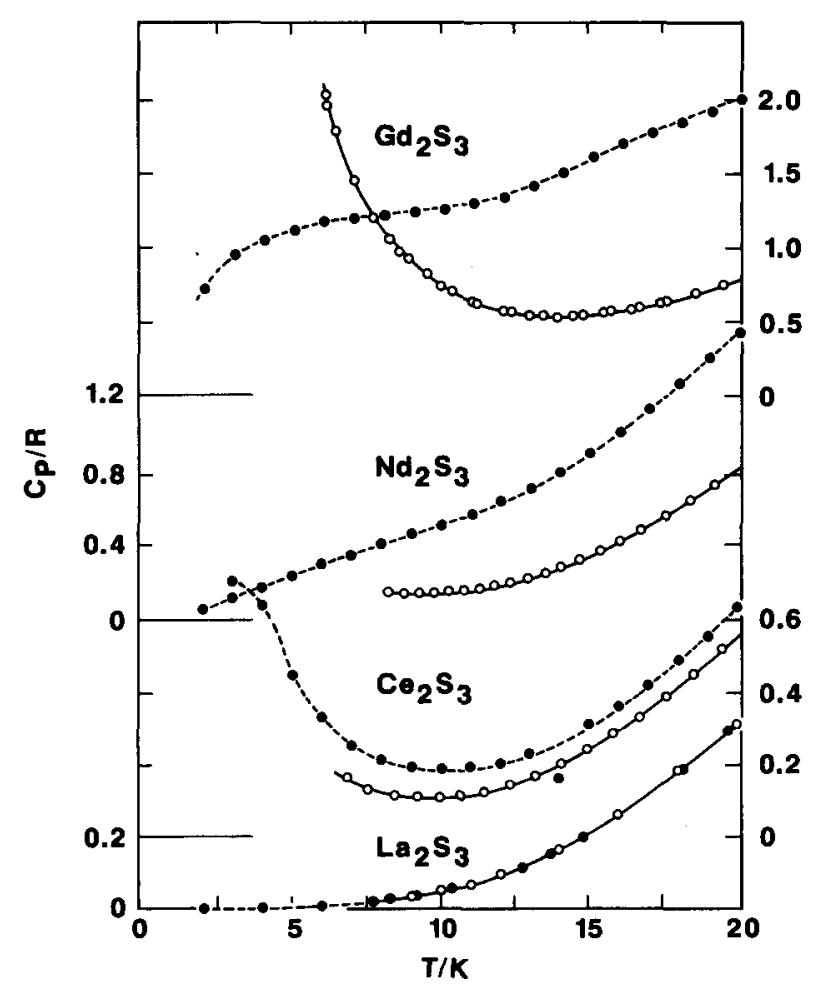

FIG. 5. Molar heat capacities as a function of temperature; present study $\left(-\mathrm{O}-\mathrm{O}-\mathrm{O}-\right.$ ); Ref. 10 (- $-1-$ ); ordinates are left $\left(\mathrm{La}_{2} \mathrm{~S}_{3}\right)$, right $\left(\mathrm{Ce}_{2} \mathrm{~S}_{3}\right)$, left $\left(\mathrm{Nd}_{2} \mathrm{~S}_{3}\right)$, and right $\left(\mathrm{Gd}_{2} \mathrm{~S}_{3}\right)$.

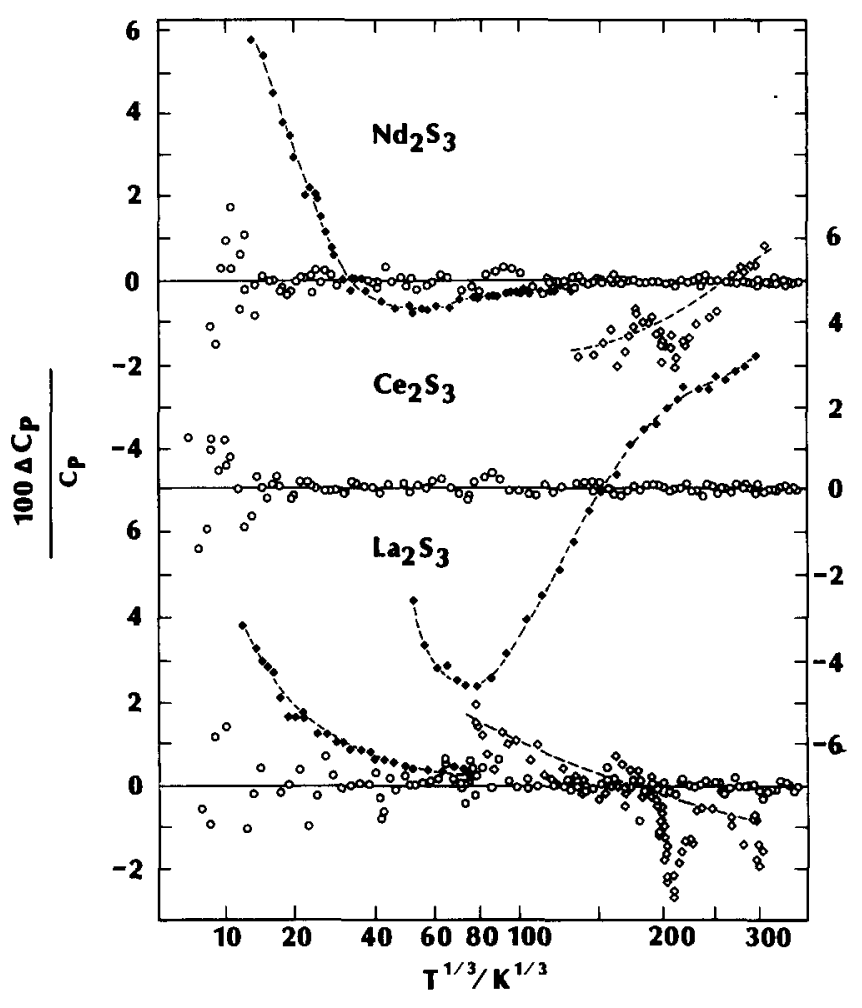

FIG. 6. Percent deviation from the smoothed curve of molar heat capacity vs temperature; ordinates are, left $\left(\mathrm{La}_{2} \mathrm{~S}_{3}\right)$, right $\left(\mathrm{Ce}_{2} \mathrm{~S}_{3}\right)$, left $\left(\mathrm{Nd}_{2} \mathrm{~S}_{3}\right)$, present study (open circles); Ref. 9 (black squares); Ref. 17 (open squares).

below about $130 \mathrm{~K}$ and $\mathrm{La}_{2} \mathrm{~S}_{3}$ below about $80 \mathrm{~K}$ have been plotted against a percent scale an order of magnitude greater than that indicated-and used-for the other points. Note that Fig. 6 represents a variation in composition over $\gamma$ phases.

The $\mathrm{Nd}_{2} \mathrm{~S}_{3}$ values of a sample of $\alpha$ and $\beta$ phases ${ }^{17}$ deviate significantly from our values on the $\gamma$ phase. The $\mathrm{Ce}_{2} \mathrm{~S}_{3}$ sample of King and Weller ${ }^{9}$ is not identified by its phase. The deviations suggest that the Schottky contributions present in their phase differ significantly from those in the $\gamma$ phase.

The measurements of $\beta$-La $\mathrm{S}_{2} \mathrm{~S}_{3}$ of Nogteva et al. ${ }^{17}$ show a significant deviation below $130 \mathrm{~K}$ from our values on $\gamma-\mathrm{La}_{2} \mathrm{~S}_{3}$ roughly similar to their deviation on the $\mathrm{Nd}_{2} \mathrm{~S}_{3}$ data. Moreover, their values are several percent lower than ours near 200 and $300 \mathrm{~K}$. The newer data by the same authors ${ }^{18}$ on a $\gamma-\mathrm{La}_{2} \mathrm{~S}_{3}$ sample are in such remarkable accord with our data, that to avoid confusion on a figure already dense with points, we have elected only to note that our smoothed curves are in coincidence from 20 to $300 \mathrm{~K}$ by amounts well within $\pm 0.2 \%$. Their sample had a composition of $\mathrm{La}_{1.000 \pm 0.001} \mathrm{~S}_{1.481 \pm 0.005} \mathrm{O}_{0.019 \pm 0.005}$ and consisted of light yellow, optically clear crystals several $\mathrm{mm}$ on each edge. Above $40 \mathrm{~K}$, the mean deviation of the experimental points was $<0.06 \%$. Their $298.15 \mathrm{~K}$ value of $S^{0}=19.47 R$ corresponds to our value of $19.51 R$. The trend of the data indicates that their significant deviation from stoichiometry is compensated for by the oxygen present.

For the calculation of the spectroscopic Schottliy con- 
tributions to the heat capacity, we adopted the levels (and degeneracies) from Table $\mathrm{V}$ as follows:

for $\mathrm{Nd}_{2} \mathrm{~S}_{3}, 0(2), 76(2), 150(2), 180(2)$, and $385(2) \mathrm{cm}^{-1}$, for $\mathrm{Ce}_{2} \mathrm{~S}_{3}, 0(2), 185(2)$, and $358(2) \mathrm{cm}^{-1}$.

Hence, comparison with Fig. 1 shows that Schottky levels make significant contributions to the heat capacity (for $\mathrm{Ce}_{2} \mathrm{~S}_{3}$ and $\mathrm{Nd}_{2} \mathrm{~S}_{3}$-about $6 \%$ and $3 \%$ at $300 \mathrm{~K}$ ) and consequently to the thermophysical functions. On the other hand, it is evident that such small deviations as appear between spectroscopic and heat-capacity curves (cf. Figs. 2 and 3 ) are well within the experimental errors of heat-capacity and spectroscopic determinations.

From the analysis of the heat capacities of the four lanthanide sesquisulfides, we have been able to calculate the lattice contribution and hence resolve the excess contribution in excellent accord with the interpretation of the spectra of $\mathrm{La}_{2} \mathrm{~S}_{3}, \mathrm{Ce}_{2} \mathrm{~S}_{3}$, and $\mathrm{Nd}_{2} \mathrm{~S}_{3}$. The Schottky heat-capacity contributions that result from the population of the Stark levels compare well with the excess experimental heat capacity over the lattice contribution and preclude the existence of any other possible contribution to the heat capacities between 20 and $350 \mathrm{~K}$. Hence, any magnetic transitions in these samples must take place below $7 \mathrm{~K}$. Magnetic transition temperatures reported in the literature between 25 and $39 \mathrm{~K}$ for $\mathrm{Gd}_{2} \mathrm{~S}_{3}$ and between 10 and $36 \mathrm{~K}$ for $\mathrm{Nd}_{2} \mathrm{~S}_{3}$ are a consequence of deviations from the ideal sesquisulfide stoichiometry of the compounds.

Future research on heat capacities below $4 \mathrm{~K}$ and magnetic susceptibility measurements within the whole temperature range would be desirable to clarify the long-range magnetic order and complement the analyses done so far.

\section{ACKNOWLEDGMENTS}

We thank Professor Magda El-Fass, Xiao-Xia Ma, and Nancy K. Mattler for assisting in the evaluation of the heat capacities and thermodynamic functions and acknowledge the help of the now deceased Professor Paolo Franzosini of the Institute of Chemical Physics, University of Pavia, Pavia, Italy, in making measurements on $\mathrm{Ce}_{2} \mathrm{~S}_{3}$. We especially appreciate consultations with and the enabling cooperation of Professor Karl A. Gschneidner, Jr., and the cooperation of Ames Laboratory personnel already mentioned. We also wish to thank Dr. J. R. Henderson for discussions and permission to report the optical spectra. J. B. G. also wishes to thank the American Society for Engineering Education for their support during the summer months. The portion of this research done at the University of Michigan was supported in part by the Structural Chemistry and Chemical Thermodynamics Program of the National Science Foundation under Grant CHE-8007977. PEP and BJB were supported in part by the U.S. Department of Energy, contract W-7405ENG-82, Ames Laboratory, Iowa State University. Support made available through the Office of Basic Energy Sciences, USDOE.

'J. R. Henderson, M. Muramoto, E. Loh, and D. M. Johnson, Purification and Growth of Rare Earth Compound Semiconductors, DAC-59368 P (McDonnell-Douglas Astronautics, Santa Monica, 1966).
${ }^{2}$ J. R. Henderson, M. Muramoto, E. Loh, and J. B. Gruber, J. Chem. Phys. 47, 3347 (1967).

${ }^{3}$ J. B. Gruber, J. R. Henderson, M. Muramoto, and E. Loh, Bull. Am. Phys. Soc. II 14, 310 (1969).

${ }^{4} J$. R. Henderson, M. Muramoto, J. B. Gruber, and R. Menzel, J. Chem. Phys. 52, 2311 (1970).

${ }^{5}$ I. E. Paukov, V. V. Nogteva, and E. I. Yarembash, Zhur. Fiz. Khim. 43, 2351 (1969); translated in Russ. J. Phys. Chem. 43, 1316 (1969).

${ }^{6} \mathrm{~J}$. M. D. Coey, B. Cornut, F. Holtzberg, and S. von Molnar, J. Appl. Phys. 50, 1923 (1979).

'S. M. A. Taher, J. B. Gruber, J. C. Ho, and D. C. Yeh, in The Rare Earths in Modern Science and Technology, edited by G. J. McCarthy and J. J. Rhyne (Plenum, New York, 1978), Vol. 1, p. 359.

${ }^{\text {}}$ K. A. Gschneidner, Jr., B. J. Beaudry, T. Takeshita, and S. S. Eucker, Phys. Rev. B 24, 7187 (1981).

${ }^{9}$ E. G. King and W. W. Weller, U.S. Bureau of Mines Bulletin, RI-5485 (1959).

${ }^{10}$ J. C. Ho, S. M. A. Taher, G. B. King, J. B. Gruber, B. J. Beaudry, and K. A. Gschneidner, Jr., J. Phys. 39, C6 (1978).

'K. A. Gschneidner, Jr., T. Takeshita, B. J. Beaudry, O. D. McMasters, S. M. A. Taher, J. C. Ho, G. B. King, and J. B. Gruber, J. Phys. Suppl. 40, C5 (1979).

${ }^{12}$ S. M. A. Taher, J. C. Ho, and J. B. Gruber, J. Chem. Phys. 76, 609 (1982).

${ }^{13}$ J. C. Ho, S. M. A. Taher, J. B. Gruber, and K. A. Gschneidner, Jr., Phys. Rev. B 26, 1369 (1982).

${ }^{14}$ S. M. Taher, J. C. Ho, J. B. Gruber, B. J. Beaudry, and K. A. Gschneidner, Jr., in The Rare Earths in Modern Science and Technology, edited by G. J. McCarthy and J. J. Rhyne (Plenum, New York, 1978), Vol. 2, p. 423.

${ }^{15}$ E. Bucher, K. Andres, F. J. Di Salvo, J. P. Maita, A. C. Gossard, A. S. Cooper, and G. W. Hull, Jr., Phys. Rev. B 11, 500 (1975).

${ }^{16}$ V. V. Tikhonov and I. A. Smirnov, Fiz. Tverd. Tela 13, 2749 (1971); translated in Sov. Phys. Solid State 13, 2296 (1971).

${ }^{17}$ V. V. Nogteva, I. E. Paukov, and E. I. Yarembash, Zhur. Fiz. Khim. 43, 2344 ( 1968); translated in Russ. J. Phys. Chem. 43, 1312 (1969).

${ }^{18}$ V. V. Nogteva, O. A. Nabutovskaya, V. N. Naumov, and V. V. Sokolov, Zhur. Fiz. Khim. 58, 2591 (1984), Russ. J. Phys. Chem. 58, 1573 (1984).

${ }^{19}$ J. B. Gruber, R. P. Leavitt, and C. A. Morrison, J. Chem. Phys. 79, 1664 (1983).

${ }^{20}$ T. Takeda, J. Magnetism Magnetic Mater. 5, 315 (1977).

${ }^{21}$ G. Becker, J. Feldhaus, K. Westerholt, and S. Methfessel, J. Magnetism Magnetic Mater. 6, 14 (1977).

${ }^{22}$ J. B. Gruber, R. D. Chirico, and E. F. Westrum, Jr., J. Chem. Phys. 76, 4600 (1982).

${ }^{23}$ E. F. Westrum, Jr., Pure Appl. Chem. 55, 539 (1983).

${ }^{24}$ J. B. Gruber, R. Burriel, E. F. Westrum, Jr., P. E. Palmer, and B. J. Beaudry, J. Less-Common Metals 94, 227 (1983).

${ }^{25}$ O. Massenet, J. M. D. Coey, and F. Holtzberg, J. Phys. 37, C4 (1977).

${ }^{26}$ L. N. Vasil'ev, A. V. Golubkov, A. G. Gorobets, V. S. Oskotskii, I. A. Smirnov, and V. V. Tikhonov, Wroclaw Conference Proceedings (1981), p. 62.

${ }^{27}$ T. Takeshita, K. A. Gschneidner, Jr., and B. J. Beaudry, J. Appl. Phys. 57, 4633 (1985).

${ }^{28} \mathrm{~B}$. J. Beaudry and K. A. Gschneidner, Jr., in Handbook on the Physics and Chemistry of Rare Earths, edited by K. A. Gschneidner, Jr. and L. Eyring (North-Holland, Amsterdam, 1978), Vol. 1, p. 173.

${ }^{29}$ American Smelting and Refining Company, Denver.

${ }^{30} \mathrm{M}$. Picon, L. Domange, J. Flahaut, M. Guittard, and M. Patrie, Bull. Soc. Chim. France 2, 221 (1960).

${ }^{31}$ E. D. West and E. F. Westrum, Jr., in Experimental Thermodynamics, edited by J. P. McCullough and D. W. Scott (Butterworths, London, 1968), Vol. 1, p. 333.

${ }^{32}$ E. F. Westrum, Jr., in Thermodynamics and its Applications to Chemical and Biochemical Systems, edited by M. A. V. Ribeiro da Silva (Reidel, Dordrecht, 1984), p. 745.

${ }^{33}$ E. F. Westrum, Jr., J. Chem. Thermodyn. 15, 305 (1983).

${ }^{34}$ R. D. Chirico, E. F. Westrum, Jr., J. B. Gruber, and J. Warmkessel, J. Chem. Thermodyn. 11, 835 (1979).

${ }^{35}$ R. D. Chirico and E. F. Westrum, Jr., J. Chem. Thermodyn. 12, 71 (1980).

${ }^{36}$ R. D. Chirico, E. F. Westrum, Jr., and J. B. Gruber, J. Chem. Thermodyn. 12, 311 (1980).

${ }^{37}$ R. D. Chirico and E. F. Westrum, Jr., J. Chem. Thermodyn. 13, 519 (1981).

${ }^{38}$ R. D. Chirico and E. F. Westrum, Jr., J. Chem. Thermodyn. 13, 1087 (1981). 
${ }^{39} \mathrm{~N}$. Komada and E. F. Westrum, Jr. (manuscript in preparation).

${ }^{40}$ V. P. Zhuze, A. A. Kamarzin, M. G. Karin, K. K. Sidorin, and A. I Shelykh, Fiz. Tverd. Tela 21, 3410 (1979); translated in Sov. Phys. Solid State 21, 1968 (1979).

${ }^{4}$ 'T. G. Arkatova, V. P. Zhuze, M. G. Karin, A. A. Kamarzin, A. A. Kuk- harskii, B. A. Mikhailov, and A. I. Shelykh, Sov. Phys. Solid State 21, 1979 (1979).

${ }^{42}$ J. R. Henderson, M. Muramoto, and J. B. Gruber, unpublished data, 1969. 